\title{
On the characterization of claw-free graphs with given total restrained domination number
}

\author{
Xiaoming $\mathrm{Pi}^{*}$
}

\section{*Correspondence:} pixiaoming1977@hotmail. com

Department of Mathematics, Harbin Normal University, Harbin 150025, China

\begin{abstract}
A set $S$ of vertices in graph $G=(V, E)$ is a total restrained dominating set, abbreviated TRDS, of $G$ if every vertex of $G$ is adjacent to a vertex in $S$ and every vertex of $V-S$ is adjacent to a vertex in $V-S$. The total restrained domination number of $G$, denoted by $\gamma_{t r}(G)$, is the minimum cardinality of a TRDS of $G$. Jiang and Kang $(J$ Comb Optim. 19:60-68, 2010) characterized the connected claw-free graph $G$ of order $n$ with $\gamma_{t r}(G)=n$. This paper studies the total restrained domination number of claw-free graphs and characterizes the connected claw-free graph $G$ of order $n$ with $\gamma_{t r}(G)=n-2$.
\end{abstract}

Keywords: Claw-free graphs, Total restrained dominating set, Total restrained domination number

Mathematics Subject Classification (2000): 05C69

\section{Background}

Let $G=(V, E)$ be a simple graph with vertex set $V$ and edge set $E$. For a vertex $u$ of $V$, let $N_{G}(u)=\{v \in V \mid u v \in E\}$ denote the open neighborhood of $u$ and $N_{G}[u]=N(u) \cup\{u\}$ denote the closed neighborhood of $u$. The degree of $u$ is denoted by $d_{G}(u)$ (briefly $N(u)$, $N[u], d(u)$ when no ambiguity on the graph is possible). For a subset $S$ of $V$, let $G[S]$ be the subgraph of $G$ induced by $S$. A vertex $v$ is called a support vertex of $G$ if $v$ is adjacent to a vertex of degree one. A support vertex is strong if it is adjacent to at least two vertices of degree one. Let $S(G)$ and $L(G)$ denote the set of all support vertices and all vertices of degree one in $G$, respectively. An edge is called a pendant edge if it is incident with a vertex of degree one. Let $\omega(G)$ denote the number of components of $G$. The corona $H \circ K_{1}$ of a graph $H$ is the graph obtained from $H$ by attaching a pendant edge to each vertex of $H$. A cycle of order $k$ is denoted by $C_{k}$. A graph is claw-free if it contains no $K_{1,3}$ as an induced subgraph. A set $S$ of vertices in graph $G=(V, E)$ is a total restrained dominating set, abbreviated TRDS, of $G$ if every vertex of $G$ is adjacent to a vertex in $S$ and every vertex of $V-S$ is adjacent to a vertex in $V-S$. The total restrained domination number of $G$, denoted by $\gamma_{t r}(G)$, is the minimum cardinality of a TRDS of G. A TRDS of cardinality $\gamma_{t r}(G)$ is called a $\gamma_{t r}$-set. For other notations and graph theory terminologies we in general follow Haynes et al. (1998).

(c) 2016 The Author(s). This article is distributed under the terms of the Creative Commons Attribution 4.0 International License (http://creativecommons.org/licenses/by/4.0/), which permits unrestricted use, distribution, and reproduction in any medium, provided you give appropriate credit to the original author(s) and the source, provide a link to the Creative Commons license, and indicate if changes were made. 
The concept of total restrained domination in graphs was introduced in Haynes et al. (1998), albeit indirectly, as a vertex partitioning problem and has been studied, in Telle and Proskurowski (1997), Cyman and Raczek (2006), Dankelmann et al. (2006), Hattingh et al. (2007), Henning and Maritz (2008), Ma et al. (2005), Raczek (2007), Raczek and Cyman (2008), Zelinka (2005) and Jiang and Kang (2010). Jiang and Kang (2010) characterized the connected claw-free graph $G$ of order $n$ with $\gamma_{t r}(G)=n$.

This paper characterizes the connected claw-free graph $G$ of order $n$ with $\gamma_{t r}(G)=n-2$.

\section{Main results}

Lemma 1 (Jiang and Kang 2010) Let $G$ be a connected claw-free graph with order $n \geq 2$. Then $\gamma_{t r}(G)=n$ if and only if $G \in \Gamma$, where $\Gamma=\cup_{i=0}^{3} \Gamma_{i}, \Gamma_{0}=\{G \mid G$ is the corona $K_{m} \circ K_{1}$ of $\left.K_{m}, m \geq 1\right\}, \Gamma_{1}$ is a collection of all graphs obtained from $G^{\prime} \in \Gamma_{0}$ by subdividing exactly one pendant edge, $\Gamma_{2}$ is a collection of all graphs obtained from $G^{\prime} \in \Gamma_{0}$ by adding a new vertex and joining it to all the support vertices of $G^{\prime}$, and $\Gamma_{3}$ is a collection of all graphs obtained from $G_{1}, G_{2} \in \Gamma_{0}\left(\left|V\left(G_{1}\right)\right|,\left|V\left(G_{2}\right)\right| \geq 3\right)$ by adding a new vertex $u$ and joining it to all the support vertices of graphs $G_{1}$ and $G_{2}$.

For completing our characterization, we define a family $\Gamma^{2}$ of claw-free graphs as follows.

$\Gamma_{0}^{2}$ is a collection of all graphs obtained from $G_{1}, G_{2} \in \Gamma_{0}$ by joining $G_{1}, G_{2}$ with an edge $u v$, where $u \in L\left(G_{1}\right), v \in L\left(G_{2}\right)$ and $\left|V\left(G_{1}\right)\right|,\left|V\left(G_{2}\right)\right| \geq 4$.

$\Gamma_{1}^{2}$ is a collection of all graphs obtained from $G_{1}, G_{2} \in \Gamma_{0}$ by joining $G_{1}, G_{2}$ with a path $P_{3}=(u, v, x)$, where $u \in L\left(G_{1}\right), x \in L\left(G_{2}\right),\left|V\left(G_{1}\right)\right|,\left|V\left(G_{2}\right)\right| \geq 4$.

$\Gamma_{2}^{2}$ is a collection of all graphs obtained from $G_{1} \in \Gamma_{0}-\left\{K_{2}\right\}$ and $K_{3}$ by adding a new edge $u v$, where $u \in L\left(G_{1}\right)$ and $v \in V\left(K_{3}\right)$.

$\Gamma_{3}^{2}$ is a collection of all graphs obtained from $G_{1}, G_{2} \in \Gamma_{2}$ by joining $G_{1}, G_{2}$ with an edge $u v$, where $u \in V\left(G_{1}\right)-S\left(G_{1}\right)-L\left(G_{1}\right), v \in V\left(G_{2}\right)-S\left(G_{2}\right)-L\left(G_{2}\right)$ and $\left|V\left(G_{1}\right)\right|,\left|V\left(G_{2}\right)\right| \geq 5$.

$\Gamma_{4}^{2}$ is a collection of all graphs obtained from $G_{1}, G_{2} \in \Gamma_{0}$ by uniting two vertices $u$ and $v$, where $u \in L\left(G_{1}\right), v \in L\left(G_{2}\right)$ and $\left|V\left(G_{1}\right)\right|,\left|V\left(G_{2}\right)\right| \geq 4$.

$\Gamma_{5}^{2}$ is a collection of all graphs obtained from $G^{\prime} \in \Gamma-\Gamma_{0}$ by attaching a pendant edge to a vertex of $L\left(G^{\prime}\right)$, where $\left|V\left(G^{\prime}\right)\right| \geq 5$.

$\Gamma_{6}^{2}$ is a collection of a 5-cycle, a 6-cycle, a 7-cycle and all graphs obtained from $G^{\prime} \in \Gamma_{0}$ by adding an edge $u v$, where $u, v \in L\left(G^{\prime}\right)$ and $\left|V\left(G^{\prime}\right)\right| \geq 4$.

$\Gamma_{7}^{2}$ is a collection of all graphs obtained from $G^{\prime} \in \Gamma-\left(\Gamma_{0} \cup\left\{P_{3}\right\}\right)$ by adding a new vertex and joining it to every vertex of $V\left(G^{\prime}\right)-L\left(G^{\prime}\right)$.

$\Gamma_{8}^{2}$ is a collection of all graphs obtained from $G_{1} \in \Gamma_{2} \cup \Gamma_{3}, G_{2} \in \Gamma_{0}$ by deleting a vertex $u$ of $L\left(G_{1}\right)$ and joining $u^{\prime}$ to every vertex of $S\left(G_{2}\right)$, where $u^{\prime} \in N_{G_{1}}(u)$ and $\left|V\left(G_{1}\right)\right| \geq 5$. $\Gamma_{9}^{2}$ is a collection of all graphs obtained from $G^{\prime} \in \Gamma_{1}$ by adding a new vertex and joining it to every vertex of $N[u]$, where $u$ is a 2-degree vertex in $S\left(G^{\prime}\right)$.

$\Gamma_{10}^{2}$ is a collection of all graphs obtained from $G_{1}, G_{2} \in \Gamma_{0}$ by adding a new vertex and joining it to each endpoint of a pendant edge of $G_{1}$ and every vertex of $S\left(G_{2}\right)$ or from 
$G_{1} \in \Gamma_{0}, K_{2}$ by adding a new vertex and joining it to each endpoint of a pendant edge of $G_{1}$ and one vertex of $K_{2}$.

$\Gamma_{11}^{2}$ is a collection of all graphs obtained from $G^{\prime} \in \Gamma_{0}$ by adding a new vertex and joining it to every vertex of $S\left(G^{\prime}\right)$ and a vertex of $L\left(G^{\prime}\right)$, where $\left|V\left(G^{\prime}\right)\right| \geq 4$.

$\Gamma_{12}^{2}$ is a collection of all graphs obtained from $G^{\prime} \in \Gamma_{1}$ by adding a new vertex and joining it to each endpoint of a pendant edge of $G^{\prime}$.

Let $\Gamma^{2}=\cup_{i=0}^{12} \Gamma_{i}^{2}$

Theorem 1 Let $G$ be a connected claw-free graph of order $n \geq 4$. Then $\gamma_{t r}(G)=n-2$ if and only if $G \in \Gamma^{2}$.

Proof Clearly when $G \in \Gamma^{2}, \gamma_{t r}(G)=n-2$. Let $G$ be a connected claw-free graph of order $n$ with $\gamma_{t r}(G)=n-2$ and $S$ be a $\gamma_{t r}$-set of $G$. Let $V-S=\left\{v_{1}, v_{2}\right\}$. Clearly, $G[S]$ has at most three components.

Claim 1 Let $C$ be a component of $G[S]$. If $N\left(v_{i}\right) \cap V(C)=\left\{v^{\prime}\right\}, i=1,2$, then $G\left[N_{C}\left[v^{\prime}\right]\right] \simeq K_{d_{C}\left(v^{\prime}\right)}$.

Proof It is obvious.

Claim 2 Let $C, C^{\prime}$ be two components of $G[S]$. If $d\left(v_{1}\right)=d\left(v_{2}\right)=2$, $N\left(v_{1}\right) \cap V(C)=\left\{v^{\prime}\right\}$ and $N\left(v_{2}\right) \cap V\left(C^{\prime}\right)=\left\{v^{\prime \prime}\right\}$, then $G \in \Gamma_{0}^{2} \cup \Gamma_{1}^{2} \cup \Gamma_{2}^{2} \cup \Gamma_{4}^{2} \cup \Gamma_{5}^{2}$.

Proof Let $C, C^{\prime} \neq K_{2}$. Then by Claim $1, \quad v^{\prime} \notin S(C), v^{\prime \prime} \notin S\left(C^{\prime}\right)$. Denote $G^{\prime}=G\left[V(C) \cup\left\{v_{1}\right\}\right]$ and $G^{\prime \prime}=G\left[V\left(C^{\prime}\right) \cup\left\{v_{2}\right\}\right]$. Assume that $\gamma_{t r}\left(G^{\prime}\right) \leq|V(C)|-1$ and $S^{\prime}$ is a $\gamma_{t r}$-set of $G^{\prime}$. Since $v_{1}$ is a 1-degree vertex of $G^{\prime}, v_{1}, v^{\prime} \in S^{\prime}$. Consequently $S^{\prime} \cup\left(V\left(C^{\prime}\right)-\left\{v^{\prime \prime}\right\}\right)$ is a TRDS of $G$, which is a contradiction. Hence $\gamma_{t r}\left(G^{\prime}\right)=|V(C)|+1$ and by Lemma $1, G^{\prime} \in \Gamma$. By the same reason, $G^{\prime \prime} \in \Gamma$. Obviously, $G^{\prime}, G^{\prime \prime} \notin \Gamma_{2} \cup \Gamma_{3}$. Let $G^{\prime} \in \Gamma_{1}$. Assume that $d\left(v^{\prime}\right) \neq 2$. Then $G^{\prime} \neq P_{5}$. Let $u$ be the 2-degree vertex in $S\left(G^{\prime}\right)$ and $u^{\prime}$ be the neighbor of $u$ not in $L\left(G^{\prime}\right)$. Then $V(G)-\left\{u^{\prime}, v^{\prime}, v_{1}\right\}$ is a TRDS of $G$, which is a contradiction. Hence $d\left(v^{\prime}\right)=2$. By the same reason, when $G^{\prime \prime} \in \Gamma_{1}, d\left(v^{\prime \prime}\right)=2$. Clearly, at most one of $G^{\prime}, G^{\prime \prime}$ is in $\Gamma_{1}$. When $G^{\prime}, G^{\prime \prime} \in \Gamma_{0}, G \in \Gamma_{0}^{2}$. When one of $G^{\prime}, G^{\prime \prime}$ is in $\Gamma_{1}, G \in \Gamma_{1}^{2}$.

Let one of $C, C^{\prime}$ be isomorphic to $K_{2}$. Without loss of generality, let $C^{\prime}=K_{2}$. Assume that $C \notin \Gamma$ and $S^{\prime}$ is a $\gamma_{t r}$-set of $C$. Then $\left|S^{\prime}\right| \leq|V(C)|-2$. If $v^{\prime} \in S^{\prime}$, then $S^{\prime} \cup V\left(C^{\prime}\right)$ is a TRDS of $G$, which is a contradiction. Hence $v^{\prime} \notin S^{\prime}$. However, $S^{\prime} \cup V\left(C^{\prime}\right) \cup\left\{v_{2}\right\}$ is a TRDS of $G$, which is a contradiction. Thus $C \in \Gamma$. By Claim 1 , when $C \neq K_{2}, v^{\prime} \notin S(C)$. Assume that $C \in \Gamma_{3}$. By the claw-freeness of $G, v^{\prime} \in L(C)$ and $V(G)-\left\{v^{\prime}, x, y\right\}$ is a TRDS of $G$, where $x \in N_{C}\left(v^{\prime}\right)$ and $y \in V(C)-S(C)-L(C)$. It's a contradiction. So $C \notin \Gamma_{3}$. When $C \in \Gamma_{0}, v^{\prime} \in L(C)$ and $G \in \Gamma_{0}^{2} \cup\left\{P_{6}\right\} \subseteq \Gamma_{0}^{2} \cup \Gamma_{5}^{2}$. When $C \in \Gamma_{1}$, we also have $v^{\prime} \in L(C)$. Let $u \in N_{C}\left(v^{\prime}\right)$. Then $u$ is the vertex with minimum degree in $S(C)$. Therefore $G \in \Gamma_{1}^{2} \cup\left\{P_{7}\right\} \subseteq \Gamma_{1}^{2} \cup \Gamma_{4}^{2}$. When $C \in \Gamma_{2}, v^{\prime} \in V(C)-S(C)-L(C)$ and $G \in \Gamma_{2}^{2} \cup \Gamma_{4}^{2}$.

Claim 3 Let $C$ be a component of $G[S]$. If $d=\left|N\left(v_{i}\right) \cap V(C)\right| \geq 2, i=1,2$, then $G\left[N\left(v_{i}\right) \cap V(C)\right] \simeq K_{d}$. 
Proof Without loss of generality, let $d=\left|N\left(v_{1}\right) \cap V(C)\right| \geq 2$. Assume that $u_{1}, u_{2} \in N\left(v_{1}\right) \cap V(C)$ and $u_{1} u_{2} \notin E(G)$. By the claw-freeness of $G$, either $v_{2} u_{1} \in E(G)$ or $v_{2} u_{2} \in E(G)$. Without loss of generality, let $v_{2} u_{1} \in E(G)$. By the connectivity of $C$, there is a shortest path $P=\left(u_{1}=w_{1}, w_{2}, \ldots, w_{p}=u_{2}\right)(p \geq 3)$ joining $u_{1}$ to $u_{2}$ in $C$. If $u_{2} \notin S(C)$, then we get a contradiction that $S-\left\{u_{2}\right\}$ is a TRDS of $G$. Hence $u_{2} \in S(C)$. Let $u^{\prime} \in N_{C}\left(u_{2}\right) \cap L(C)$. By the claw-freeness of $G$, $v_{1} w_{p-1} \in E(G)$ or $v_{1} u^{\prime} \in E(G)$. If $v_{1} u^{\prime} \in E(G)$, then $S-\left\{u^{\prime}\right\}$ is a TRDS of $G$, which is a contradiction. Hence $v_{1} w_{p-1} \in E(G)$. By the same reason, $w_{p-1} \in S(C)$. If $w_{p-2} \in N_{C}\left(w_{p-1}\right) \cap L(C)$, then $u_{1}=w_{p-2}$ and $\left(S-\left\{u_{1}, w_{p-1}\right\}\right) \cup\left\{v_{2}\right\}$ is a TRDS of $G$, which is a contradiction. So $w_{p-2} \notin N_{C}\left(w_{p-1}\right) \cap L(C)$. Let $u^{\prime \prime} \in N_{C}\left(w_{p-1}\right) \cap L(C)$. Then $G\left[\left\{u_{2}, w_{p-1}, w_{p-2}, u^{\prime \prime}\right\}\right] \simeq K_{1,3}$, which is a contradiction. So $u_{1} u_{2} \in E(G)$ and $G\left[N\left(v_{1}\right) \cap V(C)\right] \simeq K_{d}$.

Claim 4 Let $C$ be a component of $G[S]$ and $\left|N\left(v_{1}\right) \cap V(C)\right| \geq 2$. If $\left|N\left(v_{2}\right) \cap V(C)\right|=1$, then $(1) \omega(G[S]) \leq 2$ and when $|V(C)| \geq 3, C=G[S] ;(2) C \in\left\{P_{3}\right\} \cup \Gamma_{0} \cup \Gamma_{2}-\left\{K_{3}\right\}$ and $G \in \Gamma_{6}^{2} \cup \Gamma_{9}^{2} \cup \Gamma_{11}^{2}$.

Proof Let $u \in N\left(v_{2}\right) \cap V(C)$. We affirm that $N\left(v_{1}\right)-\left\{v_{2}\right\} \subseteq V(C)$. Otherwise, by the claw-freeness $G, N\left(v_{1}\right) \cap(S-V(C)) \subseteq N\left(v_{2}\right) \cap(S-V(C))$. When $|V(C)|=2$, we get the contradiction that $(S-V(C)) \cup\left\{v_{1}\right\}$ is a TRDS of $G$; when $|V(C)| \geq 3$, by Claim 1, $u \notin S(C)$ and we get the contradiction that $S-\{u\}$ is a TRDS of $G$.

1. By the claw-freeness of $G$, at most two components of $G[S]$ contain vertices of $N\left(v_{2}\right)-\left\{v_{1}\right\}$. It follows that $\omega(G[S]) \leq 2$. Let $|V(C)| \geq 3$. Assume that $\omega(G[S])=2$ and $C^{\prime}$ is a component of $G[S]$ other than $C$. Then $N\left(\nu_{2}\right) \cap V\left(C^{\prime}\right) \neq \emptyset$. By Claim 1 and $|V(C)| \geq 3, u \notin S(C)$. Therefore $S-\{u\}$ is a TRDS of $G$, which is a contradiction. Hence $G[S]$ is connected.

2. Let $|V(C)|=2$. If $C=G[S]$, then the result holds. Assume that $\omega(G[S])=2$ and $C^{\prime}$ is the other component of $G[S]$. By above, $N\left(v_{1}\right) \cap V\left(C^{\prime}\right)=\emptyset$. If $N\left(v_{2}\right) \cap V\left(C^{\prime}\right) \nsubseteq S\left(C^{\prime}\right)$ and $u^{\prime}$ is a neighbor of $v_{2}$ in $V\left(C^{\prime}\right)-S\left(C^{\prime}\right)$, then $C^{\prime} \neq K_{2}$ and $S-\left\{u^{\prime}\right\}$ also is a TRDS of $G$, which is a contradiction. Hence $N\left(v_{2}\right) \cap V\left(C^{\prime}\right) \subseteq S\left(C^{\prime}\right)$. By the connectivity of $C^{\prime}$ and the claw-freeness of $G$, we have $V\left(C^{\prime}\right)-N\left(v_{2}\right)-L\left(C^{\prime}\right)=\emptyset$. By Claim 1 and Claim 3, $G\left[N\left(v_{2}\right) \cap V\left(C^{\prime}\right)\right]$ is a complete graph and $C^{\prime} \in \Gamma_{0}$. Clearly, $G\left[V\left(C^{\prime}\right) \cup\left\{v_{2}\right\}\right] \neq K_{3}$. Thus $G \in \Gamma_{9}^{2}$.

Let $|V(C)| \geq 3$. By (1), $C=G[S]$. Assume that $C \notin \Gamma$ and $S^{\prime}$ is a $\gamma_{t r}$-set of $C$. Clearly $\gamma_{t r}(C)=|V(C)|-2$. Let $u^{\prime}, u^{\prime \prime}$ be in $V(C)-S^{\prime}$. Then $u^{\prime} u^{\prime \prime} \in E(G)$ and at least one of $N\left(v_{1}\right) \cap S^{\prime}, N\left(v_{2}\right) \cap S^{\prime}$ is empty. If $N\left(v_{1}\right) \cap S^{\prime}=\emptyset$ and $N\left(v_{2}\right) \cap S^{\prime} \neq \emptyset$ (or $N\left(v_{1}\right) \cap S^{\prime} \neq \emptyset$ and $N\left(v_{2}\right) \cap S^{\prime}=\emptyset$ ), then $S^{\prime} \cup\left\{v_{2}\right\}$ (or $\left.S^{\prime} \cup\left\{v_{1}\right\}\right)$ is a TRDS of $G$, which is a contradiction. Thus $N\left(v_{1}\right) \cap S^{\prime}=N\left(v_{2}\right) \cap S^{\prime}=\emptyset$. Then $\left(N\left(v_{1}\right) \cup N\left(v_{2}\right)\right) \cap V(C)=\left\{u^{\prime}, u^{\prime \prime}\right\}$. Let $u^{\prime}=u \in N\left(v_{1}\right) \cap N\left(v_{2}\right)$. Then $S^{\prime} \cup\left\{u^{\prime}\right\}$ is a TRDS of $G$, a contradiction. So $C \in \Gamma$.

Clearly $C \neq K_{3}$. Assume that $C \in \Gamma-\left(\left\{P_{3}\right\} \cup \Gamma_{0} \cup \Gamma_{2}\right)$. By claw-freeness of $G$ and $\left|N\left(v_{2}\right) \cap V(C)\right|=1, u \in L(C)$. Let $u^{\prime} \in N_{C}(u)$ and $u^{\prime \prime} \in V(C)-S(C)-L(C)$. If $v_{1} u^{\prime} \in E(G)$, then $V(G)-\left\{v_{1}, u^{\prime}, u^{\prime \prime}\right\}$ is a TRDS of $G$, which is a contradiction. Hence $v_{1} u^{\prime} \notin E(G)$. It follows that $v_{1} u \notin E(G)$. Otherwise since $v_{1}$ has a neighbor other than $u$, 
$V(G)-\left\{v_{2}, u, u^{\prime}\right\}$ is a TRDS of $G$, which is a contradiction. If $d\left(u^{\prime}\right) \geq 3$, then we have the contradiction that $V(G)-\left\{u, u^{\prime}, u^{\prime \prime}\right\}$ is a TRDS of $G$. So $d\left(u^{\prime}\right)=2$ and $C \in \Gamma_{1}$. Assume that $v_{1}$ is adjacent to a vertex $v$ of $L(C)-\{u\}$. Then by the claw-freeness of $G, v_{1} v^{\prime} \in G$, where $v^{\prime} \in N_{C}(v)$. However, $V(G)-\left\{v, v^{\prime}, u^{\prime \prime}\right\}$ is a TRDS of $G$, a contradiction. Hence $N\left(v_{1}\right) \cap L(C)=\emptyset$. By the claw-freeness, Claim 3 and $v_{1} u^{\prime} \notin G, v_{1}$ is adjacent to every vertex of $V(C)-\left(L(C) \cup\left\{u^{\prime}\right\}\right)$. However, $V(G)-\left\{u^{\prime}, u^{\prime \prime}, v_{1}\right\}$ is a TRDS of $G$, which is a contradiction. Hence $C \in\left\{P_{3}\right\} \cup \Gamma_{0} \cup \Gamma_{2}-\left\{K_{2}, K_{3}\right\}$.

When $C=P_{3}$, it is easy to check that $G \in \Gamma_{11}^{2}$. Let $C \neq P_{3}$. Clearly $u \notin S(C)$. Let $u \in L(C)$ and $u^{\prime} \in N_{C}(u)$. Assume that $C \in \Gamma_{2}-\left\{K_{3}\right\}$. Let $v^{\prime}$ be the vertex in $V(C)-S(C)-L(C)$. Then $V(G)-\left\{u, u^{\prime}, v^{\prime}\right\}$ is a TRDS of $G$, which is a contradiction. Hence $C \in \Gamma_{0}$. (a) When $v_{1}$ is adjacent to $u$. By Claim 3, $N\left(v_{1}\right) \cap V(C)=\left\{u, u^{\prime}\right\}$ and $G \in \Gamma_{9}^{2}$. (b) When $v_{1}$ isn't adjacent to $u$. If $v_{1}$ is adjacent to a vertex $v$ of $L(C)$, then by the claw-freeness of $G, v_{1}$ must be adjacent to $v^{\prime} \in N_{C}(v)$. Therefore $V(G)-\left\{v, v^{\prime}, u^{\prime}\right\}$ is a TRDS of $G$, which is a contradiction. Hence $N\left(v_{1}\right) \cap L(C)=\emptyset$ and $N\left(v_{1}\right) \cap V(C) \subseteq S(C)$. By the claw-freeness of $G, N\left(v_{1}\right) \cap V(C)=S(C)$. Therefore $G \in \Gamma_{6}^{2}$. Let $u \in V(C)-S(C)-L(C)$. Then $C \in \Gamma_{2}-\left\{K_{3}\right\}$. If $v_{1}$ is adjacent to a vertex $v$ of $L(C)$, then by Claim 3, $v_{1}$ must be adjacent to $v^{\prime} \in N_{C}(v)$. Hence $S-\{v\}$ is a TRDS of $G$, which is a contradiction. Thus $N\left(v_{1}\right) \cap V(C) \subseteq V(C)-L(C)$. By the claw-freeness of $G$ and $\left|N\left(v_{1}\right) \cap V(C)\right| \geq 2, N\left(v_{1}\right) \cap V(C)=V(C)-L(C)$ and $G \in \Gamma_{11}^{2}$.

Claim 5 Let $C, C^{\prime}$ be two components of $G[S]$. If $N\left(v_{1}\right) \cap V(C)=\left\{v^{\prime}\right\}$, $N\left(v_{1}\right) \cap V\left(C^{\prime}\right)=\emptyset, \quad N\left(v_{2}\right) \cap V(C)=\emptyset$ and $\left|N\left(v_{2}\right) \cap V\left(C^{\prime}\right)\right| \geq 2$, then $C \in \Gamma-\Gamma_{3}$, $G\left[V\left(C^{\prime}\right) \cup\left\{v_{2}\right\}\right] \in \Gamma_{2}$ and $G \in \Gamma_{0}^{2} \cup \Gamma_{1}^{2} \cup \Gamma_{2}^{2} \cup \Gamma_{4}^{2} \cup \Gamma_{5}^{2} \cup \Gamma_{12}^{2}$.

Proof If $\left|V\left(C^{\prime}\right)\right|=2$, then $C^{\prime}=K_{2}$ and $G\left[\left\{v_{2}\right\} \cup V\left(C^{\prime}\right)\right]=K_{3} \in \Gamma_{2}$. Let $\left|V\left(C^{\prime}\right)\right| \geq 3$. If there is a vertex $v$ in $N\left(v_{2}\right) \cap V\left(C^{\prime}\right)$ with degree $\left|N\left(v_{2}\right) \cap V\left(C^{\prime}\right)\right|$, then by Claims 1 and $3, S-\{v\}$ is a TRDS of $G$, which is a contradiction. Hence for every vertex $v$ of $N\left(v_{2}\right) \cap V\left(C^{\prime}\right), d(v) \geq\left|N\left(v_{2}\right) \cap V\left(C^{\prime}\right)\right|+1$. If there is a vertex $v$ in $N\left(v_{2}\right) \cap V\left(C^{\prime}\right)-S\left(C^{\prime}\right)$, then $S-\{v\}$ is a TRDS of $G$, which is a contradiction. Thus $N\left(v_{2}\right) \cap V\left(C^{\prime}\right) \subseteq S\left(C^{\prime}\right)$. By the claw-freeness of $G, N\left(v_{2}\right) \cap V\left(C^{\prime}\right)=S\left(C^{\prime}\right)$ and $G\left[V\left(C^{\prime}\right) \cup\left\{v_{2}\right\}\right] \in \Gamma_{2}$

Assume that $C \notin \Gamma$ and $S^{\prime}$ is a $\gamma_{t r}$-set of $C$. Then $\left|S^{\prime}\right| \leq|V(C)|-2$. If $v^{\prime} \in S^{\prime}$, then $S^{\prime} \cup(S-V(C))$ is a TRDS of $G$, which is a contradiction. Hence $v^{\prime} \notin S^{\prime}$. However, $S^{\prime} \cup(S-V(C)) \cup\left\{v_{2}\right\}$ is a TRDS of $G$, which also is a contradiction. Thus $C \in \Gamma$. By Claim 1, when $C \not K_{2}, v^{\prime} \notin S(C)$. Assume that $C \in \Gamma_{3}$. Then by the claw-freeness of $G$, $v^{\prime} \in L(C)$. Let $v$ be the vertex in $V(C)-S(C)-L(C)$. Let $v^{\prime \prime}$ be the common neighbor of $v^{\prime}$ and $v$, then $V(G)-\left\{v, v^{\prime}, v^{\prime \prime}\right\}$ is a TRDS of $G$, which is a contradiction. So $C \in \Gamma-\Gamma_{3}$.

By the claw-freeness of $G$, when $C \in \Gamma_{0} \cup \Gamma_{1}, v^{\prime} \in L(C)$. When $G\left[V\left(C^{\prime}\right) \cup\left\{v_{2}\right\}\right]=K_{3}$, $C \in\left\{K_{2}, P_{3}\right\} \cup\left(\Gamma_{2}-\left\{K_{3}\right\}\right)$. Otherwise, if $C \in\left(\Gamma_{0}-\left\{K_{2}\right\}\right) \cup\left(\Gamma_{1}-\left\{P_{3}\right\}\right)$ and $v^{\prime \prime}$ is the neighbor of $v^{\prime}$ in $V(C)$, then $V(G)-\left(\left\{v^{\prime}, v^{\prime \prime}\right\} \cup V\left(C^{\prime}\right)\right)$ is a TRDS of $G$, which is a contradiction; if $C=K_{3}$, then $\left\{v^{\prime}, v_{1}, v_{2}\right\}$ is a TRDS of $G$, which also is a contradiction.

Let $G[S]$ has exactly two components. When $C \in \Gamma_{0}$, if $G\left[V\left(C^{\prime}\right) \cup\left\{v_{2}\right\}\right]=K_{3}$, then $C=K_{2}$ and $G \in \Gamma_{12}^{2}$; if $G\left[V\left(C^{\prime}\right) \cup\left\{v_{2}\right\}\right] \neq K_{3}$, then when $C=K_{2}, G \in \Gamma_{5}^{2}$, and when $C \neq K_{2}, G \in \Gamma_{0}^{2}$. Let $C \in \Gamma_{1}$. If $C=P_{3}$, then clearly $C-\left\{v^{\prime}\right\} \in \Gamma_{0}$. Let $C \neq P_{3}, v^{\prime \prime}$ be the vertex in $V(C)-S(C)-L(C)$ and $v^{\prime \prime \prime}$ be the common neighbor 
of $v^{\prime}, v^{\prime \prime}$. If $C-\left\{v^{\prime}\right\} \notin \Gamma_{0}$, then $V(G)-\left\{v^{\prime}, v^{\prime \prime}, v^{\prime \prime \prime}\right\}$ is a TRDS of $G$, which is a contradiction. Hence $C-v^{\prime} \in \Gamma_{0}$. When $C=P_{3}$ and $G\left[V\left(C^{\prime}\right) \cup\left\{v_{2}\right\}\right]=K_{3}, G \in \Gamma_{2}^{2}$; when $G\left[V\left(C^{\prime}\right) \cup\left\{v_{2}\right\}\right] \neq K_{3}, \quad G \in \Gamma_{1}^{2} ; \quad$ when $\quad C \in \Gamma_{1}-P_{3}, \quad G\left[V\left(C^{\prime}\right) \cup\left\{v_{2}\right\}\right] \neq K_{3}$ (otherwise $S-\left(\left\{v^{\prime}, v^{\prime \prime \prime}\right\}\right) \cup V\left(C^{\prime}\right) \cup\left\{v_{1}, v_{2}\right\}$ is a TRDS of $\left.G\right)$ and $G \in \Gamma_{1}^{2}$. Let $C \in \Gamma_{2}$ and $v^{\prime \prime} \in V(C)-S(C)-L(C)$. If $v^{\prime} \in L(C)$ and $v^{\prime \prime \prime}$ is the common neighbor of $v^{\prime}$ and $v^{\prime \prime}$, then $V(G)-\left\{v^{\prime}, v^{\prime \prime}, v^{\prime \prime \prime}\right\}$ is a TRDS of $G$, which is a contradiction. Hence $v^{\prime}=v^{\prime \prime}$. Then when $G\left[V\left(C^{\prime}\right) \cup\left\{v_{2}\right\}\right]=K_{3}, G \in \Gamma_{2}^{2}$; when $G\left[V\left(C^{\prime}\right) \cup\left\{v_{2}\right\}\right] \neq K_{3}, G \in \Gamma_{2}^{2} \cup \Gamma_{4}^{2}$. Let $G[S]$ has the third component $C^{\prime \prime}$. Then by the claw-freeness of $G, v_{1}$ and $v_{2}$ have a common neighbor in $C^{\prime \prime}$ and $N\left(v_{1}\right) \cap V\left(C^{\prime \prime}\right)=N\left(v_{2}\right) \cap V\left(C^{\prime \prime}\right)$. If $C \neq K_{2}$, then by Claim 1 and Claim 3, $S-\left\{v^{\prime}\right\}$ is a TRDS of $G$, a contradiction. Hence $C=K_{2}$. Clearly $G\left[V\left(C^{\prime \prime}\right) \cup\left\{v_{1}, v_{2}\right\}\right] \neq K_{4}$ and $\left|V\left(C^{\prime \prime}\right)\right| \geq 3$. If there is a vertex $v^{\prime \prime} \in N\left(v_{1}\right) \cap V\left(C^{\prime \prime}\right)$ such that $d\left(v^{\prime \prime}\right)=d_{G\left[V\left(C^{\prime \prime}\right) \cup\left\{v_{1}, v_{2}\right\}\right]}\left(v_{1}\right)$, then $S-\left\{v^{\prime \prime}\right\}$ is a TRDS of $G$, which is a contradiction. Hence for any vertex $v^{\prime \prime} \in N\left(v_{1}\right) \cap V\left(C^{\prime \prime}\right), d\left(v^{\prime \prime}\right) \geq d_{G\left[V\left(C^{\prime \prime}\right) \cup\left\{v_{1}, v_{2}\right\}\right]}\left(v_{1}\right)+1$. It follows that $N\left(v_{1}\right) \cap V\left(C^{\prime \prime}\right) \subseteq S\left(C^{\prime \prime}\right)$. By the claw-freeness of $G, N\left(v_{1}\right) \cap V\left(C^{\prime \prime}\right)=S\left(C^{\prime \prime}\right)$ and $C^{\prime \prime} \in \Gamma_{0}$. Therefore when $G\left[V\left(C^{\prime}\right) \cup\left\{v_{2}\right\}\right]=K_{3}, G \in \Gamma_{12}^{2}$; when $G\left[V\left(C^{\prime}\right) \cup\left\{v_{2}\right\}\right] \neq K_{3}$, $G \in \Gamma_{5}^{2}$.

Claim 6 Let $C, C^{\prime}$ be two components of $G[S]$. If $\left|N\left(v_{1}\right) \cap V(C)\right|,\left|N\left(v_{2}\right) \cap V\left(C^{\prime}\right)\right| \geq 2$ and $\left|N\left(v_{1}\right) \cap V\left(C^{\prime}\right)\right|=\left|N\left(v_{2}\right) \cap V(C)\right|=0$, then $G\left[V(C) \cup V\left(C^{\prime}\right) \cup\left\{v_{1}, v_{2}\right\}\right] \in \Gamma_{3}^{2} \cup \Gamma_{12}^{2}$.

Proof By the same discussing as the proof of $G\left[V\left(C^{\prime}\right) \cup\left\{v_{2}\right\}\right] \in \Gamma_{2}$ in Claim 5, we have $G\left[V(C) \cup\left\{v_{1}\right\}\right], G\left[V\left(C^{\prime}\right) \cup\left\{v_{2}\right\}\right] \in \Gamma_{2}$. Clearly, at most one of $G\left[V(C) \cup\left\{v_{1}\right\}\right], G\left[V\left(C^{\prime}\right) \cup\left\{v_{2}\right\}\right]$ is isomorphic to $K_{3}$. When one of them is isomorphic to $K_{3}, G\left[V(C) \cup V\left(C^{\prime}\right) \cup\left\{v_{1}, v_{2}\right\}\right] \in \Gamma_{12}^{2}$; when neither of them is isomorphic to $K_{3}$, $G\left[V(C) \cup V\left(C^{\prime}\right) \cup\left\{v_{1}, v_{2}\right\}\right] \in \Gamma_{3}^{2}$.

Claim 7 Let $C$ be a component of $G[S], N\left(v_{1}\right) \cap V(C)=\left\{v^{\prime}\right\}$ and $N\left(v_{2}\right) \cap V(C)=\left\{v^{\prime \prime}\right\}$. If $v^{\prime}=v^{\prime \prime}$, then $G\left[V(C) \cup\left\{v_{1}, v_{2}\right\}\right] \in \Gamma_{2}^{2} \cup \Gamma_{8}^{2} \cup \Gamma_{10}^{2} \cup \Gamma_{12}^{2}$. If $\quad v^{\prime} \neq v^{\prime \prime}$, then $G\left[V(C) \cup\left\{v_{1}, v_{2}\right\}\right] \in \Gamma_{6}^{2}$.

Proof Denote $G^{\prime}=\left[V(C) \cup\left\{v_{1}, v_{2}\right\}\right]$. Let $v^{\prime}=v^{\prime \prime}$. If $C \in\left\{K_{2}, K_{3}, P_{3}\right\}$, then $G^{\prime} \in \Gamma_{10}^{2} \cup \Gamma_{12}^{2}$. Let $|V(C)| \geq 4$. Assume that $C-\left\{v^{\prime}\right\} \notin \Gamma$ and $S^{\prime}$ is a $\gamma_{t r}$-set of $C-\left\{v^{\prime}\right\}$. If $\left|S^{\prime}\right| \leq|V(C)|-4$, then $S^{\prime} \cup\left\{v^{\prime}, v_{1}, v_{2}\right\} \cup(S-V(C))$ is a TRDS of $G$, which is a contradiction. If $N_{C}\left(v^{\prime}\right) \cap S^{\prime} \neq \emptyset$, then $S^{\prime} \cup\left\{v^{\prime}\right\} \cup(S-V(C))$ is a TRDS of $G$, which is a contradiction. So $\left|S^{\prime}\right|=|V(C)|-3$ and $N_{C}\left(v^{\prime}\right) \cap S^{\prime}=\emptyset$. Therefore $d\left(v^{\prime}\right) \leq 4$. If $N\left(v^{\prime}\right)=\left\{v_{1}, v_{2}, u\right\}$, then $N(u)-\left(S^{\prime} \cup\left\{v^{\prime}\right\}\right) \neq \emptyset$. Let $u^{\prime} \in N(u)-\left(S^{\prime} \cup\left\{v^{\prime}\right\}\right)$. Then $V(G)-\left\{u, u^{\prime}, v^{\prime}\right\}$ is a TRDS of $G$, which is a contradiction. Hence $d\left(v^{\prime}\right)=4$. Therefore $N_{C}\left(v^{\prime}\right)=V(C)-\left\{v^{\prime}\right\}-S^{\prime}$. Let $N_{C}\left(v^{\prime}\right)=\left\{u, u^{\prime}\right\}$. Then $u u^{\prime} \in E(G), d(u), d\left(u^{\prime}\right) \geq 3$ and $u, u^{\prime} \notin S\left(C-\left\{v^{\prime}\right\}\right)$. Therefore $V(G)-\left\{u, u^{\prime}, v^{\prime}\right\}$ is a TRDS of $G$, which also is a contradiction. So $\gamma_{t r}\left(C-\left\{v^{\prime}\right\}\right)=|V(C)|-1$ and $C-\left\{v^{\prime}\right\} \in \Gamma$. Clearly $C-\left\{v^{\prime}\right\} \neq K_{3}$. When $C-\left\{v^{\prime}\right\}=P_{3}$, it is easy to check that $G^{\prime} \in \Gamma_{8}^{2} \cup \Gamma_{12}^{2}$. Let $C-\left\{v^{\prime}\right\} \neq P_{3}$. Let $v^{\prime}$ be adjacent to a vertex $u$ in $S\left(C-\left\{v^{\prime}\right\}\right)$. Then by the claw-freeness of $G$, either $v^{\prime}$ is adjacent to every vertex of $N_{C-\left\{v^{\prime}\right\}}(u)-L\left(C-\left\{v^{\prime}\right\}\right)$ or $N_{C}\left(v^{\prime}\right)=\left\{u, u^{\prime}\right\}$, where $u^{\prime} \in N(u) \cap L\left(C-\left\{v^{\prime}\right\}\right)$. For the former, $G^{\prime} \in \Gamma_{8}^{2} \cup \Gamma_{10}^{2} \cup \Gamma_{12}^{2}$. For the latter, $C-\left\{v^{\prime}\right\} \in \Gamma_{0}$ and $G^{\prime} \in \Gamma_{10}^{2}$. Let $v^{\prime}$ only be adjacent to a vertex in $V\left(C-\left\{v^{\prime}\right\}\right)-S\left(C-\left\{v^{\prime}\right\}\right)$. Then $d\left(v^{\prime}\right)=3$. When $v^{\prime}$ is adjacent 
to one vertex of $L\left(C-\left\{v^{\prime}\right\}\right), C-\left\{v^{\prime}\right\} \in \Gamma_{0}$ and $G^{\prime} \in \Gamma_{2}^{2}$. When $v^{\prime}$ is adjacent to the vertex in $V\left(C-\left\{v^{\prime}\right\}\right)-S\left(C-\left\{v^{\prime}\right\}\right)-L\left(C-\left\{v^{\prime}\right\}\right)$, by the claw-freeness of $G, C-\left\{v^{\prime}\right\} \in \Gamma_{2}$ and $G^{\prime} \in \Gamma_{12}^{2}$.

Let $v^{\prime} \neq v^{\prime \prime}$. Then by the claw-freeness of $G$, either $G[S]$ is connected or $G[S]$ has exactly two components $C, C^{\prime}$ and $N_{G^{\prime \prime}}\left[v_{1}\right]=N_{G^{\prime \prime}}\left[v_{2}\right]$, where $G^{\prime \prime}=G\left[V\left(C^{\prime}\right) \cup\left\{v_{1}, v_{2}\right\}\right]$. For the latter, $C=K_{2}$. Otherwise there is a vertex $v\left(\neq v^{\prime \prime}\right)$ adjacent to $v^{\prime}$. When $v^{\prime} v^{\prime \prime} \in E(G)$, by the claw-freeness of $G, v v^{\prime \prime} \in E(G)$ and $S-\left\{v^{\prime}\right\}$ is a TRDS of $G$; when $v^{\prime} v^{\prime \prime} \notin E(G)$, since $C$ is connected, there is a path $v^{\prime} v \cdots v^{\prime \prime}$ and $S-\left\{v^{\prime}\right\}$ also is a TRDS of G. By Claim 3, $G\left[N\left(v_{1}\right) \cap V\left(C^{\prime}\right)\right] \simeq K_{d_{G^{\prime \prime}}\left(v_{1}\right)-1}$. If there is a vertex $v$ in $N\left(v_{1}\right) \cap V\left(C^{\prime}\right)-S\left(C^{\prime}\right)$, then $V(C) \cup\left(V\left(C^{\prime}\right)-\{v\}\right)$ is a TRDS of $G$, which is a contradiction. Hence $N\left(v_{1}\right) \cap S\left(C^{\prime}\right) \neq \emptyset$. If $N\left(v_{1}\right) \cap V\left(C^{\prime}\right) \nsubseteq S\left(C^{\prime}\right)$, then $S-\left(\left(N\left(v_{1}\right) \cap V\left(C^{\prime}\right)-S\left(C^{\prime}\right)\right)\right.$ is a TRDS of $G$, which is a contradiction. Hence $N\left(v_{1}\right) \cap V\left(C^{\prime}\right) \subseteq S\left(C^{\prime}\right)$. Clearly $G^{\prime \prime} \neq K_{4}$. By the claw-freeness of $G, N\left(v_{1}\right) \cap V\left(C^{\prime}\right)=S\left(C^{\prime}\right)$ and $G-E(C) \in \Gamma_{0}$. Therefore $G \in \Gamma_{6}^{2}$. For the former, let $S^{\prime}$ be a $\gamma_{t r}$-set of $G-\left\{v_{1} v_{2}\right\}$. Since $S^{\prime}$ is a TRDS of $G,\left|S^{\prime}\right| \geq n-2$. By $v_{1}, v_{2} \in L\left(G-\left\{v_{1} v_{2}\right\}\right), v_{1}, v_{2}, v^{\prime}, v^{\prime \prime} \in S^{\prime}$.

Let $\left|S^{\prime}\right|=n$. Then $G-\left\{v_{1} v_{2}\right\} \in \Gamma$. When $|V(C)|=2, G=C_{4}$. When $|V(C)|=3$, $G=C_{5}$. Let $|V(C)| \geq 4$. Then $G-\left\{v_{1} v_{2}\right\} \in \Gamma_{0}$ and $G \in \Gamma_{6}^{2}$.

Let $\left|S^{\prime}\right|=n-2$. Clearly $|V(C)| \geq 4$ and at least one of $\left(N\left(v^{\prime}\right)-\left\{v_{1}\right\}\right) \cap S^{\prime},\left(N\left(v^{\prime \prime}\right)-\left\{v_{2}\right\}\right) \cap S^{\prime}$ is empty. Let only one of them be empty. Without loss of generality, let $\left(N\left(v^{\prime}\right)-\left\{v_{1}\right\}\right) \cap S^{\prime}=\emptyset$. Then $d_{C}\left(v^{\prime}\right) \leq 2$. Assume that $N_{C}\left(v^{\prime}\right)=\left\{w, w^{\prime}\right\}$. Then $w, w^{\prime} \notin S^{\prime}, w w^{\prime} \in E(G)$ and one and only one of $w, w^{\prime}$ is 2-degree. Without loss of generality, let $d(w)=2$. Then $\left(S^{\prime} \cup\left\{w^{\prime}\right\}\right)-\left\{v_{1}, v^{\prime}\right\}$ is a TRDS of $G$, which is a contradiction. Thus $d_{C}\left(v^{\prime}\right)=1$. Let $N_{C}\left(v^{\prime}\right)=\{w\}$ and $w^{\prime}$ be the vertex in $N(w)-S^{\prime}$. Then $d(w)=2$. Otherwise there is a vertex $w^{\prime \prime}\left(\neq v^{\prime}, w^{\prime}\right)$ adjacent to $w$ and $S^{\prime}-\left\{v^{\prime}\right\}$ is a TRDS of $G$. Let $u$ be any vertex in $\left(N\left(v^{\prime \prime}\right)-\left\{v_{2}\right\}\right) \cap S^{\prime}$. By Claim 1 and the connectivity of $C, d(u) \geq 2$ and when $w^{\prime} v^{\prime \prime} \in E(G), w^{\prime} u \in E(G)$. If $d\left(v^{\prime \prime}\right) \geq 4$, then there are at least two neighbors of $v^{\prime \prime}$ in $S^{\prime}$ and $S^{\prime}-\left\{v^{\prime \prime}, v_{2}\right\}$ is a TRDS of $G$, which is a contradiction. Hence $d\left(v^{\prime \prime}\right) \leq 3$. Assume that $w^{\prime} \notin N(u)$ or $w^{\prime} \in N(u)$ and $d(u) \geq 3$. Then $S^{\prime}-\left\{v_{2}, v^{\prime \prime}\right\}$ is a TRDS of $G$, which is a contradiction. Thus $N(u)=\left\{v^{\prime \prime}, w^{\prime}\right\}$. If $v^{\prime \prime} w \in E(G)$, then $\left(S^{\prime}-\left\{u, v_{2}, v^{\prime \prime}\right\}\right) \cup\left\{w, w^{\prime}\right\}$ is a TRDS of $G$, which is a contradiction. Thus $v^{\prime \prime} w \notin E(G)$ and $d\left(v^{\prime \prime}\right)=2$. If $d\left(w^{\prime}\right) \geq 3$, then by the claw-freeness of $G, d(u) \geq 3$, a contradiction. Hence $d\left(w^{\prime}\right)=2$. It follows that $G=C_{7} \in \Gamma_{6}^{2}$.

Let $\left(N\left(v^{\prime}\right)-\left\{v_{1}\right\}\right) \cap S^{\prime}=\left(N\left(v^{\prime \prime}\right)-\left\{v_{2}\right\}\right) \cap S^{\prime}=\emptyset$. Therefore $d\left(v^{\prime}\right), d\left(v^{\prime \prime}\right) \leq 3$ and $v^{\prime} v^{\prime \prime} \notin E(G)$. Assume that $N_{C}\left(v^{\prime}\right) \cap N_{C}\left(v^{\prime \prime}\right)=\{v\}$. Then $N(v)-\left\{v^{\prime}, v^{\prime \prime}\right\} \neq \emptyset$. Let $u \in N(v)-\left\{v^{\prime}, v^{\prime \prime}\right\}$. Then $S^{\prime}=V(G)-\{u, v\}$. By the claw-freeness of $G, u v^{\prime} \in E(G)$ or $u v^{\prime \prime} \in E(G)$. Whether the former or the latter holds, we will get $S^{\prime}-\left\{v^{\prime \prime}\right\}$ or $S^{\prime}-\left\{v^{\prime}\right\}$ is a TRDS of $G$, which is a contradiction. Thus either $N_{C}\left(v^{\prime}\right) \cap N_{C}\left(v^{\prime \prime}\right)=\{u, v\}$ or $N_{C}\left(v^{\prime}\right) \cap N_{C}\left(v^{\prime \prime}\right)=\emptyset$. For the former, $N[u]=N[v]=\left\{u, v, v^{\prime}, v^{\prime \prime}\right\}$. Then $V(G)-\left\{v, v_{1}, v^{\prime}\right\}$ is a TRDS of $G$, which is a contradiction. Hence the latter holds and $d\left(v^{\prime}\right)=d\left(v^{\prime \prime}\right)=2$. Let $N\left(v^{\prime}\right)=\left\{v_{1}, u\right\}$ and $N\left(v^{\prime \prime}\right)=\left\{v_{2}, u^{\prime}\right\}$. Then $\left\{u, u^{\prime}\right\}=S-S^{\prime}$ and $u u^{\prime} \in E(G)$. Clearly, $d(u)=d\left(u^{\prime}\right)=2$ and $G=C_{6}$.

Claim 8 Let $C$ be a component of $G[S]$. If $\left|N\left(v_{1}\right) \cap V(C)\right| \geq 2$ and $\left|N\left(v_{2}\right) \cap V(C)\right| \geq 2$, then $C \in \Gamma_{0}$ and $G\left[V(C) \cup\left\{v_{1}, v_{2}\right\}\right] \in \Gamma_{7}^{2}$. 
Proof If $|V(C)|=2$, then $C=K_{2} \in \Gamma_{0} \quad$ and $\quad G\left[V(C) \cup\left\{v_{1}, v_{2}\right\}\right]=K_{4} \in \Gamma_{7}^{2}$. Let $|V(C)| \geq 3$. Then by Claim 3, for every vertex $u$ of $N\left(v_{1}\right) \cap V(C)$, $d_{C}(u) \geq\left|N\left(v_{1}\right) \cap V(C)\right|$. It follows that $N\left(v_{1}\right) \cap V(C) \subseteq S(C)$. By the same reason, $N\left(v_{2}\right) \cap V(C) \subseteq S(C)$. By the claw-freeness of $G$ and the connectivity of $C$, $N\left(v_{1}\right) \cap V(C)=N\left(v_{2}\right) \cap V(C)=S(C)$. Therefore $C \in \Gamma_{0}$ and $G\left[V(C) \cup\left\{v_{1}, v_{2}\right\}\right] \in \Gamma_{7}^{2}$.

To complete the proof, we discuss the following three cases.

Case $1 G[S]$ is connected. When $d\left(v_{1}\right)=d\left(v_{2}\right)=2$, by Claim 7, $G \in \Gamma_{2}^{2} \cup \Gamma_{6}^{2} \cup \Gamma_{8}^{2} \cup \Gamma_{10}^{2} \cup \Gamma_{12}^{2}$. When only one vertex of $v_{1}, v_{2}$ has degree at least 2 , by Claim 4, $G \in \Gamma_{6}^{2} \cup \Gamma_{9}^{2} \cup \Gamma_{11}^{2}$. When $d\left(v_{1}\right), d\left(v_{2}\right) \geq 3$, by Claim $8, G \in \Gamma_{7}^{2}$.

Case $2 G[S]$ has exactly two components $C$ and $C^{\prime}$. Let $G^{\prime}=G\left[V(C) \cup\left\{v_{1}, v_{2}\right\}\right]$ and $G^{\prime \prime}=G\left[V\left(C^{\prime}\right) \cup\left\{v_{1}, v_{2}\right\}\right]$.

Case 2.1 $v_{1} v_{2}$ is a cut edge of $G$. Then $N\left(v_{1}\right) \cap N\left(v_{2}\right)=\emptyset$. By Claims 2, 5 and 6, $G \in\left(\cup_{i=0}^{5} \Gamma_{i}^{2}\right) \cup \Gamma_{12}^{2}$.

Case 2.2 $v_{1} v_{2}$ isn't a cut edge of $G$. Then there is a component of $G[S]$ such that both of $v_{1}, v_{2}$ are adjacent to at least one vertex of it. Let $C$ be such a component and $N\left(v_{1}\right) \cap V\left(C^{\prime}\right) \neq \emptyset$. By Case $1, G^{\prime} \in \Gamma_{2}^{2} \cup\left(\cup_{i=6}^{12} \Gamma_{i}^{2}\right)$. It is easy check that $G^{\prime} \notin \Gamma_{2}^{2} \cup \Gamma_{8}^{2} \cup \Gamma_{11}^{2}$.

If $\left|V\left(C^{\prime}\right)\right|=2$, then $C^{\prime}=K_{2}$ and $N\left(v_{1}\right) \cap V\left(C^{\prime}\right) \subseteq S\left(C^{\prime}\right)$. Let $\left|V\left(C^{\prime}\right)\right| \geq 3$. Obviously, for any vertex $v$ of $N\left(v_{1}\right) \cap V\left(C^{\prime}\right), d_{C^{\prime}}(v) \geq\left|N\left(v_{1}\right) \cap V\left(C^{\prime}\right)\right|$. If there is a vertex $v$ in $N\left(v_{1}\right) \cap V\left(C^{\prime}\right)-S\left(C^{\prime}\right)$, then $S-\{v\}$ is a TRDS of $G$, which is a contradiction. Hence $N\left(v_{1}\right) \cap V\left(C^{\prime}\right) \subseteq S\left(C^{\prime}\right)$. By the connectivity of $C^{\prime}$ and the claw-freeness of $G$, for any vertex $v$ of $N\left(v_{1}\right) \cap V\left(C^{\prime}\right), d_{C^{\prime}}(v)=\left|N\left(v_{1}\right) \cap V\left(C^{\prime}\right)\right|, C^{\prime} \in \Gamma_{0}$ and $N\left(v_{1}\right) \cap V\left(C^{\prime}\right)=S\left(C^{\prime}\right)$.

Case 2.2.1 $G^{\prime} \in \Gamma_{6}^{2}$. Then $d_{G^{\prime}}\left(v_{1}\right)=2$ or $d_{G^{\prime}}\left(v_{2}\right)=2$. Without loss of generality, let $N_{G^{\prime}}\left(v_{1}\right)=\left\{v_{2}, v^{\prime}\right\}$. Since $N_{G^{\prime}}\left(v_{1}\right) \cap N_{G^{\prime}}\left(v_{2}\right)=\emptyset$, by the claw-freeness of $G$, $N\left(v_{1}\right) \cap V\left(C^{\prime}\right)=N\left(v_{2}\right) \cap V\left(C^{\prime}\right)$. If $C \neq K_{2}$, then $v^{\prime} \notin S(C)$ and $S-\left\{v^{\prime}\right\}$ is a TRDS of $G$, which is a contradiction. Hence $C=K_{2}$ and $G^{\prime}=C_{4}$. Clearly $G^{\prime \prime} \nsucceq K_{4}$. When $C^{\prime} \neq K_{2}$, both of $v_{1}, v_{2}$ are adjacent to every vertex of $S\left(C^{\prime}\right)$; when $C^{\prime}=K_{2}, v_{1}, v_{2}$ is adjacent to the same vertex $v$ of $C^{\prime}$. Hence $G \in \Gamma_{6}^{2}$.

Case 2.2.2 $G^{\prime} \in \Gamma_{7}^{2}$. Then $C \in \Gamma_{0}$ and both of $v_{1}, v_{2}$ are adjacent to every vertex of $S(C)$. Clearly $G^{\prime} \neq K_{4}$. Let $N\left(v_{2}\right) \cap V\left(C^{\prime}\right)=\emptyset$. When $C^{\prime}=K_{2}, G \in \Gamma_{5}^{2} \cup \Gamma_{8}^{2}$. When $C^{\prime} \neq K_{2}$, $G^{\prime \prime} \in \Gamma_{0}$ and $G \in \Gamma_{8}^{2}$. Let $N\left(v_{2}\right) \cap V\left(C^{\prime}\right) \neq \emptyset$. When $C^{\prime}=K_{2},\left|N\left(v_{2}\right) \cap V\left(C^{\prime}\right)\right|=1$. If $N\left(v_{1}\right) \cap N\left(v_{2}\right) \cap V\left(C^{\prime}\right)=\emptyset$, then $G \in \Gamma_{6}^{2} ; \quad$ If $\quad N\left(v_{1}\right) \cap N\left(v_{2}\right) \cap V\left(C^{\prime}\right) \neq \emptyset$, then $G \in \Gamma_{7}^{2}$. When $C^{\prime} \neq K_{2}, N\left(v_{2}\right) \cap V\left(C^{\prime}\right) \subseteq S\left(C^{\prime}\right)$. By the claw-freeness of $G$, $N\left(v_{2}\right) \cap V\left(C^{\prime}\right)=S\left(C^{\prime}\right)$ and by Claim $3, G\left[\left\{v_{2}\right\} \cup V\left(C^{\prime}\right)\right] \in \Gamma_{2}$. Thus $G \in \Gamma_{7}^{2}$.

Case 2.2.3 $G^{\prime} \in \Gamma_{9}^{2}$. Then $d_{G^{\prime}}\left(v_{1}\right)=2, d_{G^{\prime}}\left(v_{2}\right)=3$ or $d_{G^{\prime}}\left(v_{1}\right)=3, d_{G^{\prime}}\left(v_{2}\right)=2$. By $G$ having exactly two components and Claim 4(1), (2), $C=K_{2}$ and by the proof of Claim 4(2), $G \in \Gamma_{9}^{2}$.

Case 2.2.4 $G^{\prime} \in \Gamma_{10}^{2}$. Then by the connectivity of $C, d_{G^{\prime}}\left(v_{1}\right)=d_{G^{\prime}}\left(v_{2}\right)=2, v_{1}, v_{2}$ have a common neighbor $v$ in $G^{\prime}$ and $C-\{v\} \in \Gamma_{0}$. Clearly $N\left(v_{2}\right) \cap V\left(C^{\prime}\right)=\emptyset$. When $C=P_{3}, G \in \Gamma_{10}^{2}$. By the claw-freeness of $G$, Claims 1 and $3, G\left[\left\{v_{1}\right\} \cup V\left(C^{\prime}\right)\right] \in \Gamma_{2} \cup P_{3}$. 
When $C=K_{3}, G\left[V\left(C^{\prime}\right) \cup\left\{v_{1}\right\}\right] \neq K_{3}$ and $G \in \Gamma_{10}^{2}$. Let $C \neq P_{3}, K_{3}$. Assume that $v$ is adjacent to each endpoint $u, u^{\prime}$ of a pendant edge of $C-\{v\}$ and $u \in S(C-\{v\})$, then $\left(S-\left\{u^{\prime}, v\right\}\right) \cup\left\{v_{1}\right\}$ is a TRDS of $G$, which is a contradiction. Hence $N(v)=\left\{v_{1}, v_{2}\right\} \cup S(C-\{v\})$ and $G \in \Gamma_{10}^{2}$.

Case 2.2.5 $G^{\prime} \in \Gamma_{12}^{2}$. Then by the connectivity of $C, d_{G^{\prime}}\left(v_{1}\right)=d_{G^{\prime}}\left(v_{2}\right)=2, v_{1}, v_{2}$ have a common neighbor $v$ in $G^{\prime}$ and $G^{\prime}-\left\{v_{2}\right\} \in \Gamma_{1}$. Assume that $G^{\prime}-\left\{v_{2}\right\} \neq P_{3}$. Let $v^{\prime} \in V(G)-S(G)-L(G)$. Then $V(G)-\left\{v, v_{2}, v^{\prime}\right\}$ is a TRDS of $G$, which is a contradiction. Hence, $G^{\prime}-\left\{v_{2}\right\}=P_{3}$ and $v \in S\left(G^{\prime}-\left\{v_{2}\right\}\right)$. If $N\left(v_{2}\right) \cap V\left(C^{\prime}\right)=\emptyset$, then $G\left[V\left(C^{\prime}\right) \cup\left\{v_{1}\right\}\right] \in\left\{P_{3}, K_{3}\right\} \cup \Gamma_{2}$ and $G \in \Gamma_{5}^{2} \cup \Gamma_{8}^{2}$. Let $N\left(v_{2}\right) \cap V\left(C^{\prime}\right) \neq \emptyset$. When $C^{\prime}=K_{2},\left|V\left(C^{\prime}\right) \cap N\left(v_{2}\right)\right| \leq 1$. If $N\left(v_{1}\right) \cap N\left(v_{2}\right) \cap V\left(C^{\prime}\right)=\emptyset$, then $G \in \Gamma_{6}^{2}$. If $N\left(v_{1}\right) \cap N\left(v_{2}\right) \cap V\left(C^{\prime}\right) \neq \emptyset$, then $\left|N\left(v_{1}\right) \cap V\left(C^{\prime}\right)\right|=1$ and $G \in \Gamma_{7}^{2}$. Let $C^{\prime} \neq K_{2}$. Then by the claw-freeness of $G$ and Claim 3, $N\left(v_{2}\right) \cap V\left(C^{\prime}\right)=S\left(C^{\prime}\right)$ and $G^{\prime \prime} \in \Gamma_{2}$. Therefore $G \in \Gamma_{7}^{2}$.

Case $3 G[S]$ has three components $C_{1}, C_{2}$ and $C_{3}$. By the claw-freeness of $G$, both $v_{1}$ and $v_{2}$ are adjacent to at least one vertex of exactly two components of $G[S]$. Without loss of generality, let $N\left(v_{1}\right) \cap V\left(C_{1}\right) \neq \emptyset \neq N\left(v_{1}\right) \cap V\left(C_{2}\right)$ and $N\left(v_{2}\right) \cap V\left(C_{2}\right) \neq \emptyset \neq N\left(v_{2}\right) \cap V\left(C_{3}\right)$. By similarly discussing to the proof of $C^{\prime} \in \Gamma_{0}$ in Case 2.2, we have $C_{1}, C_{3} \in \Gamma_{0}$ and when $C_{1}=K_{2}\left(\right.$ or $\left.C_{3}=K_{2}\right), N\left(v_{1}\right) \cap V\left(C_{1}\right) \subseteq S\left(C_{1}\right)$ (or $\left.N\left(v_{2}\right) \cap V\left(C_{3}\right) \subseteq S\left(C_{3}\right)\right)$; when $C_{1} \neq K_{2}\left(\right.$ or $\left.C_{3} \neq K_{2}\right), N\left(v_{1}\right) \cap V\left(C_{1}\right)=S\left(C_{1}\right)$ (or $\left.N\left(v_{2}\right) \cap V\left(C_{3}\right)=S\left(C_{3}\right)\right)$. By the claw-freeness of $G, N\left(v_{1}\right) \cap N\left(v_{2}\right) \neq \emptyset$.

Case 3.1 $N\left(v_{1}\right) \cap N\left(v_{2}\right)=\left\{v^{\prime}\right\}$. Assume that $C_{2} \neq K_{2}$. Then $v^{\prime} \notin S\left(C_{2}\right)$ and $S-\left\{v^{\prime}\right\}$ is a TRDS of $G$, which is a contradiction. Hence $C_{2}=K_{2}$. Clearly $G\left[V\left(C_{1}\right) \cup\left\{v_{1}\right\}\right]$ and $G\left[V\left(C_{3}\right) \cup\left\{v_{2}\right\}\right]$ can't be isomorphic to $K_{3}$ at the same time. Let one of them be isomorphic to $K_{3}$. Without loss of generality, let $G\left[V\left(C_{1}\right) \cup\left\{v_{1}\right\}\right] \cong K_{3}$. If $C_{3}=K_{2}$, then $G \in \Gamma_{12}^{2}$; if $C_{3} \neq K_{2}$, then $G \in \Gamma_{8}^{2}$. Let neither of them be isomorphic to $K_{3}$. If one of $C_{1}, C_{3}$ is $K_{2}$, then $G \in \Gamma_{5}^{2}$; if neither of $C_{1}, C_{3}$ is $K_{2}$, then $G \in \Gamma_{8}^{2}$.

Case 3.2 $\left|N\left(v_{1}\right) \cap N\left(v_{2}\right)\right| \geq 2$. By Claim 8, $C_{2} \in \Gamma_{0}$ and $G\left[V\left(C_{2}\right) \cup\left\{v_{1}, v_{2}\right\}\right] \in \Gamma_{7}^{2}$. Clearly $C_{2} \neq K_{2}$ and $G\left[V\left(C_{1}\right) \cup\left\{v_{1}\right\}\right], G\left[V\left(C_{3}\right) \cup\left\{v_{2}\right\}\right]$ can't be isomorphic to $K_{3}$ at the same time. By similarly discussing as Case 3.1, we have when one of them is isomorphic to $K_{3}, G \in \Gamma_{8}^{2} \cup \Gamma_{12}^{2}$ and when neither of them is isomorphic to $K_{3}, G \in \Gamma_{5}^{2} \cup \Gamma_{8}^{2}$.

\section{Conclusions}

The study focuses on the total restrained domination in claw-free graphs. Firstly, in the course of analysis, I construct 12 kinds of connected claw-free graphs with order $n$ and the total restrained domination number $n-2$. Let $\Gamma^{2}$ denote the set of these clawfree graphs. Secondly, by discussing all possible cases of the induced subgraph of the minimum total restrained dominating set, I show that if the total restrained domination number of a connected claw-free graph with order $n$ is $n-2$, then the graph must belong to $\Gamma^{2}$. In a word, as for a connected claw-free graph with order $n$, the conclusion gives a method to judge whether the total restrained domination number of it is $n-2$. Further research can focus on the construction of connected claw-free graphs with order $n$ and total restrained domination number $n-3$ and characterize these graphs, although it may be very difficult and complicated. 


\section{Acknowledgements}

The work was supported by Doctoral Scientific Research Fund of Harbin Normal University (No. KGB201008).

\section{Competing interests}

The author declare that he has no competing interests.

Received: 2 December 2015 Accepted: 26 September 2016

Published online: 07 October 2016

\section{References}

Cyman J, Raczek J (2006) On the total restrained domination number of a graph. Australas J Comb 36:91-100

Dankelmann P, Hattingh JH, Henning MA, Swart HC (2006) Trees with equal domination and restrained domination numbers. J Glob Optim 34:597-607

Hattingh JH, Jonck E, Joubert EJ, Plummer AR (2007) Total restrained domination in trees. Discrete Math 307:1643-1650

Haynes TW, Hedetniemi ST, Slater PJ (1998) Fundamentals of domination in graphs. Dekker, New York

Henning MA, Maritz JE (2008) Total restrained domination in graphs with minimum degree two. Discrete Math 308:1909-1920

Jiang H, Kang L (2010) Total restrained domination in claw-free graphs. J Comb Optim 19:60-68

Ma D, Chen X, Sun L (2005) On total trastrained domination in graphs. Czechoslov Math J 55:165-173

Raczek J (2007) Trees with equal restrained domination and total restrained domination numbers. Discuss Math Graph Theory 27:83-91

Raczek J, Cyman J (2008) Total restrained domination number of trees. Discrete Math 308:44-50

Telle JA, Proskurowski A (1997) Algorithms for vertex partitioning problems on partial k-trees. SIAM J Disctrete Math 10:529-550

Zelinka B (2005) Remarks on restrained domination and total restrainec domination in graphs. Czechoslov Math 55:393-396

\section{Submit your manuscript to a SpringerOpen ${ }^{\circ}$ journal and benefit from:}

- Convenient online submission

\section{- Rigorous peer review}

- Immediate publication on acceptance

- Open access: articles freely available online

- High visibility within the field

Retaining the copyright to your article

Submit your next manuscript at $\gg$ springeropen.com 